\title{
Guideline for the optimal use of blood cultures
}

\author{
Ntobeko Ntusi, Lindsey Aubin, Stephen Oliver, Andrew Whitelaw, Marc Mendelson
}

The incidence of sepsis is increasing globally, with high morbidity and mortality. Prompt, accurate detection of bacteraemia and fungaemia is imperative for improving patient care, yet health care professionals lack training in correct blood culture techniques. These guidelines discuss the clinical importance of blood cultures, the indications for their use and the correct technique for optimal yield of pathogenic micro-organisms that cause sepsis.

S Afr Med J 2010; 100: 839-843.
Detection of bacteraemia or fungaemia by blood culture is critical in managing patients with infection, and directs the appropriate selection of antimicrobials. Blood culture is a common laboratory investigation where blood is inoculated into culture medium and incubated. Media used in blood culture bottles support the growth of most medically important bacteria and fungi, including anaerobes, which grow adequately in the aerobic blood culture bottle, hence separate anaerobic bottles are infrequently used. ${ }^{1,2}$ The goal of culturing blood is to determine whether a pathogenic microorganism(s) is responsible for the patient's clinical presentation. 'False positive' results occur when a skin commensal(s) rather than a true pathogenic organism is grown. Proper cleaning of the skin is a vital component of venesection to reduce the false-positive rate, yet poor technique is common when collecting blood for culture. This lapse results in inappropriate use of antibiotics, increased antibiotic resistance and prolonged hospital stay. A further barrier to correct laboratory interpretation of blood culture results is inadequate relevant clinical details on the accompanying request form. These guidelines contextualise the place of blood cultures in the management of sepsis and detail the appropriate method for acquiring blood for culture, to reduce the false-positive rate.

\section{Methods}

We performed a literature review of the diagnosis and management of sepsis; identified a number of international best practice standards, including recommendations of the United Kingdom Department of Health, International Sepsis Forum, Society of Critical Care Medicine,

Cardiac Clinic, Department of Medicine, Groote Schuur Hospital and University of Cape Town

Ntobeko Ntusi, FCP (SA)

Clinical Skills and Training Unit, Department of Medicine, Groote Schuur Hospital and University of Cape Town

Lindsey Aubin, RN, RM, Pg Dip Educator of Adults

Division of Microbiology, Clinical Laboratory Services, Groote Schuur Hospital and University of Cape Town

Stephen Oliver, MB ChB, MMed Path (Microbiol)

Andrew Whitelaw, MB BCh, MSc, FCPath (Microbiol) (SA)

Division of Infectious Diseases \& HIV Medicine, Department of Medicine, Groote Schuur Hospital and University of Cape Town

Marc Mendelson, PhD, FRCP (UK), DTM\&H
Surgical Infection Society, American Association of Critical Care Nurses, American Association of Chest Physicians, American College of Emergency Physicians, American Thoracic Society, Australian and New Zealand Intensive Care Society, European Society of Intensive Care Medicine, Indian Society of Critical Care Medicine, European Respiratory Society, Infectious Diseases Society of America, and the Clinical and Laboratory Standards Institute; and studied consensus expert opinion documents.

\section{Definitions}

Blood culture. Collection and inoculation of blood into culture medium with the aim of growing pathogenic bacteria or fungi for diagnostic purposes.

Bacteraemia. The presence of viable bacteria in the bloodstream which may be transient (e.g. following dental procedures), intermittent (e.g. undrained abscesses), or continuous (e.g. endovascular infection).

Fungaemia. The presence of viable fungi in the bloodstream.

Infection. An inflammatory response to one or more microorganisms, or the invasion of normally sterile sites by those organisms.

Systemic inflammatory response syndrome (SIRS). A consequence of the host inflammatory response that can follow infection or other injury, defined as the clinical cluster of two or more of:

- temperature $>38^{\circ} \mathrm{C}$ or $<36^{\circ} \mathrm{C}$

- heart rate $>90$ beats/minute

- respiratory rate $>20$ breaths/minute or $\mathrm{paCO}_{2}<4.3 \mathrm{kPa}(32$ $\mathrm{mmHg}$ )

- white cell count $>12000$ cells $/ \mathrm{mm}^{3},<4000$ cells $/ \mathrm{mm}^{3}$, or $>10 \%$ immature white blood cells (band forms). ${ }^{3}$

Sepsis. SIRS resulting from documented infection. ${ }^{3,4}$

Severe sepsis. Documented sepsis associated with organ dysfunction, hypoperfusion or hypotension. Perfusion abnormalities may manifest as, but are not limited to:

- lactic acidosis

- oliguria

- acute alteration in mental state

- areas of mottled skin

- capillary refilling requiring $\geq 3$ seconds

- disseminated intravascular coagulation

- acute lung injury or acute respiratory distress syndrome (ARDS)

- cardiac dysfunction, as defined by echocardiography or direct measurement of the cardiac index. ${ }^{3,5}$

Septic shock. The presence of severe sepsis plus one or both of the following: 
- systemic mean blood pressure $<60 \mathrm{mmHg}$ (or $<80 \mathrm{mmHg}$ if the patient has baseline hypertension) despite adequate fluid resuscitation

- maintaining systemic mean blood pressure $>60 \mathrm{mmHg}$ (or $>80 \mathrm{mmHg}$ if the patient has baseline hypertension) requires support with inotropic/vasoactive agents. ${ }^{3,4}$

Multi-organ dysfunction/failure. Multi-organ failure is often a consequence of severe sepsis and septic shock, and refers to the presence of altered function affecting more than one organ in an acutely ill patient such that homeostasis cannot be maintained without intervention. Multiple organ dysfunction syndrome (MODS) is either primary (directly attributable to a well-defined insult) or secondary (as a consequence of the host response). MODS is characterised by, but not limited to, abnormalities in serum creatinine, platelet count, serum bilirubin, Glasgow coma score, pressure adjusted heart rate, and arterial oxygenation. ${ }^{3,5}$

\section{Sepsis and the significance of blood culture}

Some 1.8 million cases of sepsis occur worldwide each year; ${ }^{6}$ however, owing to variable definitions and reporting, this is probably an underestimate. A more accurate estimate may be as high as 18 million, an incidence of $3 / 1000$ population. ${ }^{7}$ Sepsis is the most common cause of death in non-coronary intensive care units worldwide. ${ }^{8,9}$ The mortality rate from sepsis is generally between $30 \%$ and $70 \%$, and is higher in persons with a pre-existing disease. ${ }^{3,8}$ Elderly, critically ill and immunocompromised persons are particularly vulnerable. ${ }^{10}$ Population-based studies of incidence and prevalence of sepsis in South Africa have not been undertaken.

There are several reviews of the pathophysiology of sepsis. ${ }^{11-16}$ The direct effects of invading micro-organisms, their toxic products, or both, may contribute to pathogenesis of sepsis. Important factors include endotoxin, bacterial cell wall components (peptidoglycan, muramyl dipeptide, and lipoteichoic acid), and bacterial products such as staphylococcal enterotoxin B, toxic shock syndrome toxin-1, pseudomonas exotoxin $\mathrm{A}$, and $\mathrm{M}$ protein of haemolytic group $\mathrm{A}$ streptococci. ${ }^{17}$ Cellular injury and organ dysfunction occur as a consequence of ischaemia, ${ }^{18}$ cytopathic injury ${ }^{19}$ and increased rate of apoptosis. ${ }^{20}$ Panendothelial activation in sepsis also leads to widespread tissue oedema, endothelial dysfunction, hypotension, upregulation of adhesion molecules, and impaired anticoagulant properties leading to MODS. ${ }^{21}$

Blood culture plays an integral role in the evaluation of sepsis. ${ }^{22}$ As bacteraemia is often associated with fever, clinicians are encouraged to obtain blood samples for culture from febrile patients. ${ }^{23}$ The indications for blood culture are broad and ill-defined. ${ }^{24}$ Other than pyrexia, clinical parameters alone are unhelpful in predicting bacteraemia and cannot be used in isolation to determine the timing of blood sampling for culture. ${ }^{25}$

\section{Clinical features of sepsis}

The clinical features of sepsis relate to the causative organism, the involved site, severity, and the host response to infection. Symptoms and signs of sepsis are usually superimposed on the patient's underlying illness and primary infection. Hence, their rate of development and clinical presentation varies. Common clinical features of sepsis include lethargy, fever (although hypothermia may occur) and an elevated or reduced white blood cell count. ${ }^{26}$ Sweating, rigors, tachycardia, hypotension, tachypnoea, warm peripheries, confusion, oliguria and jaundice may also occur (Table I).

\section{Diagnosis of sepsis}

Sepsis is primarily a clinical diagnosis, supported by laboratory investigation and imaging. Culture of specimens from a sterile site is the gold standard microbiological investigation and the key to successful diagnosis. ${ }^{27,28}$ Occasionally, serology may aid diagnosis. Culture is a slow process compared with rapid molecular diagnostic tests that detect genetic material and can be used at the 'point-ofcare.29 A point-of-care test for sepsis would be an extremely useful addition to the diagnostic armory.

The indications for blood culture are shown in Table II. Although only $5-15 \%$ of blood cultures drawn in febrile patients are positive, approximately half of all patients with severe sepsis demonstrate bacteraemia at the time of diagnosis. ${ }^{30}$ The presence or absence of a positive blood culture does not appear to influence outcome of sepsis, suggesting that prognosis is more related to severity of sepsis. ${ }^{31}$ Hospital-acquired infections (HAI) diagnosed from blood culture have a higher mortality than those acquired in the community. ${ }^{32}$

Table I. Clinical and laboratory manifestations of sepsis

\begin{tabular}{lll}
\hline Symptoms & Signs & Laboratory findings \\
\hline Rigors & Temperature increase or decrease & Leucocytosis or leucopenia \\
Sweating & Tachycardia & Hypoalbuminaemia \\
Nausea and vomiting & Hypotension & $\begin{array}{l}\text { Increased INR, increased APTT, decreased } \\
\text { platelets, DIC }\end{array}$ \\
Symptoms related to affected organ system & Tachypnoea, hyperventilation (acute lung \\
(e.g. cough, diarrhoea, dysuria, etc.) & injury) & Hyperglycaemia \\
& Warm pink peripheries & Hyperlactataemia \\
& Confusion & Increasingly negative base excess \\
& Oliguria & Elevated CRP, ESR, ${ }^{*}$ procalcitonin, fibrinogen \\
& Jaundice & \\
& Haemorrhagic lesions, cutaneous bullae, \\
& cellulitis & \\
& & \\
*ESR should not be used in patients with HIV, as hypergammaglobulinaemia and immune hyperactivation commonly leads to an increased ESR in the absence of sepsis.
\end{tabular}


Table II. Indications for blood culture

1. Clinical features of sepsis including tachycardia, tachypnoea, increased or sub-normal temperature and change in sensorium, hypotension or prostration

2. Suspicion of infective endocarditis

3. Pyrexia of unknown origin

4. Unexplained leucocytosis or leucopenia

5. Systemic and localised infections including suspected meningitis, osteomyelitis, septic arthritis, acute untreated bacterial pneumonia or other possible bacterial infection

\section{Principles of sample collection for blood culture}

1. Blood cultures should be drawn when there is a clinical suspicion of bloodstream infection. ${ }^{33}$

2. Blood should not be collected from indwelling arterial or venous lines unless an infected intravenous line is suspected. ${ }^{34}$ Should blood be drawn for culture from an indwelling line, a second specimen should be obtained from a peripheral site.

3. Two or more blood specimens should be collected using sterile technique at separate sites, before administering antibiotics. ${ }^{35-37}$ Except in very unusual cases, no more than 3 sets of blood cultures should be collected in one 24 -hour period. If all 4 sets are negative after 24 hours and sepsis is still suspected, more cultures may be collected..$^{38} \mathrm{~A}$ larger number of cultures may have to be collected from persons already receiving antimicrobials ${ }^{39}$ although, if clinical condition allows, stopping antibiotics and re-culturing after 48 hours is preferred.

4. Ideally, a minimum of $10 \mathrm{ml}$ of blood, depending on the blood culture system used, should be inoculated into a culture bottle when taking blood from adults. ${ }^{40}$ Adequate volumes of blood improve detection of pathogenic organisms and reduce time to detection.

5. Arterial blood culture provides no advantage over venous samples. ${ }^{41}$

6. Changing needles between venepuncture and inoculation into culture bottles is controversial. Discontinuing this practice owing to lack of evidence that it decreases contamination and the increased risk of sharp injuries has been recommended. ${ }^{42,43}$ However, a meta-analysis challenges this recommendation, suggesting a slight overall benefit of switching needles. ${ }^{44}$ Until more definitive studies are available, we recommend that the risk of changing needles after venepuncture does not outweigh the benefit. More important ways to decrease contamination of blood culture bottles include the use of tincture of iodine as a disinfectant, ${ }^{45}$ avoidance of drawing blood through existing intravenous lines ${ }^{34}$ and disinfecting the membrane of the blood culture bottle. ${ }^{46}$

7. It is a medico-legal requirement that the patient's notes must always document acquiring blood for culture, including indication, site, time of taking blood culture and a legible name.

Table III details recommendations for how to correctly send blood to the laboratory for culture.

\section{Optimal number of blood cultures}

The optimal number of blood cultures that should be obtained in a febrile patient varies according to the suspected diagnosis or clinical condition, the suspicion of underlying infection, and the urgency of the need for treatment. ${ }^{47}$
- One blood culture is rarely, if ever, sufficient or advisable. ${ }^{21} \mathrm{~A}$ positive result on a single culture is difficult to interpret, unless an unequivocal pathogen is isolated.

- Two blood cultures are usually adequate when continuous bacteraemia is anticipated. ${ }^{48}$

- Three blood cultures are reasonable when intermittent bacteraemia is suspected. ${ }^{49}$

We believe that more than 3 blood cultures is rarely justifiable in a resource-limited setting. Furthermore, the probability of obtaining a false-positive result increases steadily as more blood cultures are performed.

\section{Timing of blood cultures}

Few systematic studies have been conducted on the timing of blood cultures and the optimal interval between successive tests. ${ }^{38}$ The ideal is to collect blood at time intervals ranging from one to several hours. ${ }^{50}$ However, it is sufficient and appropriate to obtain blood from 2 separate sites within minutes of each other from patients who are acutely ill or those in whom the likelihood of continuous bacteraemia is high. ${ }^{51}$ By contrast, in patients suspected to have intermittent bacteraemia, it is advisable to obtain multiple blood cultures 6 - 36 hours apart. ${ }^{38,52}$ One study showed no difference in yield whether blood samples for cultures performed within a 24-hour period were drawn simultaneously or at spaced intervals. ${ }^{53}$ Antibiotics should not be withheld in sick patients apart from a reasonable delay to collect more than 1 culture sample.

\section{Volume of blood to be taken for culture}

A minimum of $10 \mathrm{ml}$ (and preferably $20 \mathrm{ml}$ ) of blood should be obtained from adults, ${ }^{38,41,53,54}$ and $1-5 \mathrm{ml}$ (and preferably more) from infants and children. ${ }^{40,55,56}$ The yield from blood culture depends upon the volume of blood cultured, ${ }^{57-59}$ the yield in adults increasing approximately $3 \%$ per $\mathrm{ml}$ of blood cultured.

Maintaining the correct ratio of blood to culture medium is important. Most blood culture bottles contain the anticoagulant sodium polyanetholsulphonate (SPS). SPS can inactivate lysozyme, some antibiotics, and parts of the complement cascade. However, SPS can also be toxic to certain fastidious organisms. Adding sufficient blood to the bottle helps negate this latter effect. A dilution of blood in broth by a ratio of 1:5 has been recommended. ${ }^{60,61}$

\section{Type of blood culture bottle}

Most blood culture systems are now automated, with some form of continuous monitoring to detect growth. The bottles contain broth supplemented with additives. Different bottles are designed either to be used in different patient groups, or to isolate different classes of pathogens. A few general principles apply:

- Standard aerobic bottles are suitable for the recovery of most common bacterial pathogens, including aerobes and facultative anaerobes. These are not as suitable for supporting the growth of strict anaerobes, and therefore traditional advice has been to use a paired set of aerobic and anaerobic bottles when taking blood cultures. ${ }^{62}$ The routine use of anaerobic bottles has been questioned, as the incidence of anaerobic bacteraemia is decreasing ${ }^{61,63,64}$ and most infections involving anaerobes are clinically suspected and appropriate empiric therapy is administered before blood culture results become available. ${ }^{65,66}$ Conversely, improved yield of microorganisms when using aerobic-anaerobic paired blood cultures has been demonstrated. ${ }^{67,68}$ Although firm evidence is unavailable, we believe that the routine use of anaerobic bottles is not warranted, particularly in resource-limited settings.

- Some manufacturers produce aerobic and anaerobic bottles with 


\section{Table III. Procedure for blood culture}

\begin{tabular}{|c|c|}
\hline Step & Procedure \\
\hline 1 & $\begin{array}{l}\text { Verify the patient's identity: Ask the patient for his or her name. Check the armband. Look on wall above the bed or in the patient } \\
\text { notes to confirm identity. }\end{array}$ \\
\hline 2 & Inform the patient of your intentions and explain the procedure. Always obtain verbal consent. \\
\hline 3 & $\begin{array}{l}\text { Assemble the correct materials required for blood culture: } \\
\text { - blood culture bottle(s) } \\
\text { - syringe }(10 \mathrm{ml} \text { or more }) \\
\text { - needle ( } 22 \text { gauge or more) } \\
\text { - sterile gloves } \\
\text { - tourniquet } \\
\text { - adhesive strip } \\
\text { - povidone iodine or alcohol solution (or other suitable skin disinfectant) } \\
\text { - sterile pack containing cotton/gauze swabs, sterile paper x2 and waste bag } \\
\text { - patient labels } \\
\text { - sharps waste disposal bin. }\end{array}$ \\
\hline 4 & $\begin{array}{l}\text { Open the sterile blood culture pack onto the trolley. Remove the sterile sheet and place under the patient's arm. Pour povidone iodine } \\
\text { or alcohol solution into fluid recess located on blood culture tray/pack. Drop needle and syringe onto sterile field. }\end{array}$ \\
\hline 5 & $\begin{array}{l}\text { Apply tourniquet and select a suitable vein. Wash hands with soap and water or disinfect with alcohol hand disinfectant. Dry your } \\
\text { hands or rub the hand disinfectant in until dry. Apply sterile gloves. }\end{array}$ \\
\hline 6 & $\begin{array}{l}\text { Clean the puncture site with povidone or alcohol solution using aseptic technique. Allow } 1 \text { - } 2 \text { minutes for the disinfectant to dry. } \\
\text { Place green sterile cover with opening over site for blood culture. }\end{array}$ \\
\hline 7 & $\begin{array}{l}\text { Carefully insert needle into patient's blood vessel and collect a minimum of } 10 \mathrm{ml} \text { of blood (adults). If using the vacutainer system, } \\
\text { the blood culture must be the first blood specimen to be collected. }\end{array}$ \\
\hline 8 & $\begin{array}{l}\text { Release tourniquet. Remove needle and syringe from puncture site. Place dry swab on puncture site and apply pressure. If blood has } \\
\text { not been collected directly into the blood culture bottle with the vacutainer system, inoculate blood into culture bottle, having first } \\
\text { disinfected the top of the blood culture bottle with an alcohol swab (a webcol is adequate) }{ }^{50} \text { If blood is being collected for other tests, } \\
\text { always inoculate blood culture bottle first. Do not change needles between blood sample collection and inoculation of blood culture } \\
\text { bottle. }\end{array}$ \\
\hline 9 & Gently rotate the blood culture bottle to mix the blood and culture medium (do not shake vigorously). \\
\hline 10 & $\begin{array}{l}\text { Label the blood culture bottle, making sure not to remove the 'tear-off' label on the bottle. Make sure that patient labels do not cover } \\
\text { the blood culture bottle bar code label and are not stuck across the bottom of the blood culture bottle. Complete a laboratory request } \\
\text { form. Remember to include the site, date and time of collection, full clinical information regarding the suspected diagnosis, and } \\
\text { contact details for the clinician responsible for the patient. }\end{array}$ \\
\hline 11 & $\begin{array}{l}\text { Deliver the blood culture bottle to the laboratory as soon as possible. If there is a delay in getting the sample to the laboratory, do not } \\
\text { refrigerate the bottle; rather leave it at room temperature. }\end{array}$ \\
\hline
\end{tabular}

additives (such as charcoal or resins) that inactivate antibiotics. These are preferable when taking blood cultures from patients already on antimicrobials, but are more expensive. There is evidence that the average time to positivity with these bottles is shorter than with normal aerobic and anaerobic bottles; however, their cost-effectiveness is unclear. ${ }^{61}$ Paediatric bottles are often supplemented with growth factors and usually have additives which will bind antibiotics, allowing improved recovery of bacteria. Together with the lower concentration of SPS, these features allow smaller volumes of blood to be inoculated. ${ }^{60,61}$

- Mycobacterial blood culture bottles contain broth suitable for the isolation of mycobacteria and are valuable for the detection of disseminated mycobacterial infections, both $M$. tuberculosis and non-tuberculous mycobacteria. However, these bottles are often also used to enhance the recovery of yeasts from blood, although there is less evidence to justify this practice. ${ }^{69,70}$ Given the additional expense, routine blood culture bottles should be used for detection of candidaemia, and mycobacterial blood cultures should be reserved for suspected mycobacterial infections. ${ }^{71}$

\section{Conclusion}

Blood culture remains one of the most important microbiological tests available to the clinician. Members of the healthcare team who perform blood cultures must have a sound comprehension of the principles that underlie this important diagnostic tool. These guidelines provide practical and accessible guidance for medical students, nurses and doctors on the correct performance of blood collection for culture and should be applicable to all levels of healthcare in South Africa. Adoption of these guidelines and implementation of procedures to improve the quality of blood culture investigations will reduce the risk of blood sample contamination and improve the management and care of patients with sepsis.

These guidelines are endorsed by the South African Society for Clinical Microbiology (SASCM).

The authors acknowledge Dr Colleen Bamford and Professors Janet Seggie, Vanessa Burch and Bongani Mayosi for their constructive comments during the development of this document.

Dr Ntusi receives support from the Discovery Foundation and the Medical Research Council of South Africa. Professor Mendelson receives support from PEPFAR USAID through the ANOVA Health Institute. 


\section{References}

1. Murray PR, Traynor P, Hopson D. Critical assessment of blood culture techniques: analysis of recovery of obligate and facultative anaerobes, strict aerobic bacteria, and fungi in aerobic and anaerobic blood culture bottles. J Clin Microbiol 1992;30:1462-1468.

2. Morris AJ, Wilson ML, Mirrett S, Reller LB. Rationale for selective use of anaerobic blood cultures. J Clin Microbiol 1993;31:2110-2113.

3. American College of Chest Physicians/Society of Critical Care Medicine. Consensus Conference Definitions for sepsis and organ failure and guidelines for use of innovative therapies in sepsis. Crit Care Med 1992;20:864-874.

4. Levy MM, Fink MP, Marshall JC, et al. 2001 SCCM/ESCIM/ACCP/ATS/SIS International Sepsis Definitions Conference. Crit Care Med 2003;31:1250-1256

5. Balk RA. Severe sepsis and septic shock. Definitions, epidemiology, and clinical manifestations. Crit Care Clin 2000;16:179-192.

6. Angus DC, Linde-Zwirble WT, Lidicker J, et al. Epidemiology of sepsis in the US: Analysis of incidence, outcome, and associated costs of care. Crit Care Med 2001;29:1303-1310

7. Martin GS, Mannino DM, Eaton S, Moss M. The epidemiology of sepsis in the United States from 1979 through 2000. N Engl J Med 2003;348:1546-1554.

8. Vincent JL, Sakr Y, Sprung CL, et al. Sepsis in European intensive care units: results of the SOAP study. Crit Care Med 2006;34:344-353.

9. Padkin A, Goldfrad C, Brady AR, et al. Epidemiology of severe sepsis occurring in the first 24 hours in intensive care units in England, Wales, and Northern Ireland. Crit Care Med 2003;31:2332-2338.

10. Dellinger RP, Carlet JM, Masur H, et al. Surviving Sepsis Campaign guidelines for management of severe sepsis and septic shock. Crit Care Med 2004;32: 858-873.

11. Remick DG. Pathophysiology of sepsis. Am J Pathol 2007;170:1435-1444.

12. Bone RC. Immunologic dissonance: A continuing evolution in our understanding of the systemic inflammatory response syndrome (SIRS) and the multiple organ dysfunction syndrome (MODS). An Intern Med 1996:125:680-687.

13. Bone RC. The pathogenesis of sepsis. Ann Intern Med 1991;115:457-469.

14. Peters K, Unger RE, Brunner J, Kirkpatrick CJ. Molecular basis of endothelial dysfunction in sepsis. Cardiovasc Res 2003;60:49-57.

15. Rigato $\mathrm{O}$, Salomao R. Impaired production of interferon-gamma and tumor necrosis factor-alpha but not of interleukin-10 in whole blood of patients with sepsis. Shock 2003;19:113-116.

16. Pinsky MR, Matuschak GM. Multiple organ failure: Failure of host defense homeostasis. Crit Care Clin 1989;5:199-220

17. Pugin J. Recognition of bacteria and bacterial products by host immune cells in sepsis. In: Vincent IL, ed. Yearbook of Intensive Care and Emergency Medicine. Berlin: Springer-Verlag, 1996:11.

18. Piagnerelli M, Boudjeltia KZ, Vanhaeverbeek M, Vincent JL. Red blood cell rheology in sepsis. Intensive Care Med 2003;29:1052-1061.

19. Crouser ED, Julian MW, Blaho DV, Pfeiffer DR. Endotoxin-induced mitochondrial damage correlates with impaired respiratory activity. Crit Care Med 2002;30:276-284.

20. Marshall JC, Watson RW. Apoptosis in the resolution of systemic inflammation. In: Vincent JL, ed. Yearbook of Intensive Care and Emergency Medicine. Berlin: Springer-Verlag, 1997:100

21. Aird WC. The role of the endothelium in severe sepsis and multiple organ dysfunction syndrome. Blood 2003;101:3765-3777.

22. Shanson DC. Blood culture technique: current controversies. J Antimicrob Chemother 1990;25(Suppl C): $17-29$.

23. Aronson M, Bor D. Blood cultures. Ann Intern Med 1987;106:246-253.

24. Chandrasekar PH, Brown WJ. Clinical issues of blood cultures. Arch Intern Med 1994;154:841-849.

25. Peduzzi P, Shatney C, Sheagren J, Sprung C. Predictors of bacteremia and gram-negative bacteremia in patients with sepsis. Veterans Affairs Systemic Sepsis Cooperative Study Group. Arch Intern Med 1992;152:529-535.

26. Harris RL, Musher DM, Bloom K, et al. Manifestations of sepsis. Arch Int Med 1987;147:1895-1906.

27. von Landenberg P, Shoenfeld Y. New approaches in the diagnosis of sepsis. Isr Med Ass J 2001;3:439 442.

28. Cohen J. Microbiological requirements for studies of sepsis. In: Sibbald WJ, Vincent JL, eds. Clinical Trials for the Treatment of Sepsis. Berlin: Springer-Verlag, 1995:29.

29. Cursons RT, Jeyerajah E, Sleigh JW. The use of polymerase chain reaction to detect septicemia in critically ill patients. Crit Care Med 1999;27:937-940.

30. Bone RC, Fisher CJ, Clemmer TP, et al. Sepsis syndrome: A valid clinical entity. Methylprednisolone Severe Sepsis Study Group. Crit Care Med 1989;17:389-393.

31. Brun-Buisson C, Doyon F, Carlet J, et al. Bacteremia and severe sepsis in adults: A multicenter prospective survey in ICUs and wards of 24 hospitals. French Bacteremia-Sepsis Study Group. Am J Resp Crit Care Med 1996;154:617-624.

32. Shorr AF, Tabak YP, Killian AD, et al. Healthcare-associated bloodstream infection: A distinct entity? Insights from a large U.S. database. Crit Care Med 2006;34:2588-2595.

3. Henke PK, Polk HC Jr. Efficacy of blood cultures in the critically ill surgical patient. Surgery 1996;120:752-758.

34. Norberg A, Christopher NC, Ramundo ML, et al. Contamination rates of blood cultures obtained by dedicated phlebotomy vs. intravenous catheter. JAMA 2003;289:726-729.

35. Bates DW, Goldman L, Lee TH. Contamination blood cultures and resource utilization. The true consequences of false-positive results. JAMA 1991;265:365-369.

36. McKenzie R, Reimer LG. Effect of antimicrobials on blood cultures in endocarditis. Diagn Microbiol Infect Dis 1987;8:165-172.

37. Washington JA II, Ilstrup DM. Blood cultures. Issues and controversies. Rev Infect Dis 1986;8:792802

38. Weinstein MP. Current blood culture methods and systems: Clinical concepts, technology and interpretation of results. Clin Infect Dis 1996;23:40-46.
39. Pazin GJ, Saul S, Thompson ME. Blood culture positivity: suppression by outpatient antibiotic therapy in patients with bacterial endocarditis. Arch Intern Med 1982;142:263-268.

Connell TG, Rele M, Cowley D, et al. How reliable is a negative blood culture result? Volume of blood submitted for culture in routine practice in a children's hospital. Pediatrics 2007;119:891.

1. Reller LB, Murray PR, MacLowry JD. Blood Cultures II. In: Washington JA II, Cumitech IA, eds. Washington DC: American Society for Microbiology,1982.

42. Krumholz HM, Cummings S, York M. Blood culture phlebotomy: switching needles does not preven contamination. Ann Intern Med 1990;113:290-292.

43. Leisure MK, Moore DM, Schwartzman JD, et al. Changing the needle when inoculating blood cultures: A no benefit and high risk procedure. JAMA 1990;264:2111-2112.

44. Spitalnic SJ, Woolard RH, Mermel LA. The significance of changing needles when inoculating blood cultures: A meta-analysis. Clin Infect Dis 1995;21:1103-1106.

45. Little JR, Murray PR, Traynor PS, Spitznagel E. A randomized trial of povidone-iodine compared with tincture for venipuncture site disinfection: Effects on rates of blood culture contamination. Am J Med 1999;107:119-125

46. Eskira S, Gilad J, Schlaeffer P, et al. Reduction of blood culture contamination rate by an educational intervention. Clin Microbiol Infect 2006;12:818-821.

47. Lee A, Mirrett S, Reller LB, Weinstein MP. Detection of blood stream infections in adults: how many blood cultures are needed? J Clin Microbiol 2007;45:3546-3548.

48. Weinstein MP, Reller LB, Murphy JR, Lichtenstein KA. The clinical significance of positive blood cultures: a comprehensive analysis of 500 episodes of bacteremia and fungemia in adults. I. Laboratory and epidemiologic observations. Rev Infect Dis 1983;5:35-53.

49. Washington JA II. Blood cultures: principles and techniques. Mayo Clin Proc 1975;50:91-98.

50. Noskin GA, Phair JP. Approach to the patient with bacteremia and sepsis. In: Kelly WN, Dupont HL Glick JH, et al., eds. Textbook of Internal Medicine. Wickford RI, USA: Lippincott-Raven Publishers, 1997:1575-1581.

51. Strand CL. Blood cultures: consensus recommendations in 1988. Microbiology no. MB 88-1. American Society for Clinical Pathologists Check Sample Continuing Education Program. Chicago: American Society for Clinical Pathologists, 1988.

52. Ilstrup DM, Washington JA II. The importance of volume of blood cultures in the detection of bacteremia and fungaemia. Diagn Microbiol Infect Dis 1983;1:107-110.

53. Li J, Plorde JJ, Carlson LG. Effects of volume and periodicity on blood cultures. J Clin Microbio 1994:32:2829-2831.

54. Mermel LA, Maki D. Detection of bacteremia in adults: Consequences of culturing an inadequate volume of blood. Ann Intern Med 1993;119:270-272.

55. Schelonka RL, Chai MK, Yoder BA, et al. Volume of blood required to detect common neonatal pathogens. J Pediatr 1996;129:275-278

56. Brown DR, Kutler D, Rai B, et al. Bacterial concentration and blood volume required for a positive blood culture. J Perinatol 1995;15:157-159.

57. Isaacman DJ, Karasic RB, Reynolds EA, Kost SI. Effect of number of blood cultures and volume of blood on detection of bacteremia in children. J Pediatr 1996;128:190-195.

58. Plorde JJ, Tenover FC, Carlson LG. Specimen volume versus yield in the BACTEC blood culture system. J Clin Microbiol 1985;22:292-295.

59. Tenney JH, Reller LB, Mirrett S, Wang W-LL, Weinstein MP. Controlled evaluation of the volume of blood cultured in detection of bacteremia and fungemia. J Clin Microbiol 1982:15:558-561.

60. O'Hara CM, Weinstein MP, Miller JM. Manual and automated systems for detection and identification of microorganisms. In: Murray PR, Baron EJ, Jorgensen JH, Pfaller MA, Yolken RH, eds. Manual of Clinical Microbiology, 8th ed. Washington DC: ASM Press, 2003.

61. Reimer LG, Wilson ML, Weinstein MP. Update on detection of bacteraemia and fungaemia. Clin Microbiol Rev 1997:10:444-465.

62. Mylotte JM, Tayara A. Blood cultures: clinical aspects and controversies. Eur J Clin Microbiol Infect Dis 2000;10:157-163.

63. Lazarovitch T, Freimann S, Shapira G, Blank H. Decrease in anaerobe-related bacteraemias and increas in Bacteroides species isolation rate from 1998 to 2007: a retrospective study (in press). Anaerobe 2009 doi:10.1016/j.anaerobe.2009.09.003

64. Dorsher CW, Rosenblatt JE, Wilson WR, Ilstrup DM. Anaerobic bacteremia: decreasing rate over 15year period. Rev Infect Dis 1991;3:633-636.

65. Iwata K, Takahashi M. Is anaerobic blood culture necessary? If so, who needs it? Am J Med Sci 2008; $336: 58-63$

66. Ciobutaro P, Lishner M, Kilan A, et al. Decreasing the use of anaerobic blood culture bottles in selected febrile patients - is it reasonable? Eur I Intern Med 2005:16:485-488.

67. Karunakaran R, Raja NS, Quek KF, et al. Evaluation of the routine use of the anaerobic bottle when using the BACTEC blood culture system. I Microbiol Immunol Infect 2007;40:445-449.

68. Riley JA, Heiter BJ, Bourbeau PP. Comparison of recovery of blood culture isolates from two BacT/ ALERT FAN aerobic blood culture bottles with recovery from one FAN aerobic bottle and one FAN anaerobic bottle. J Clin Microbiol 2003:41:213-217.

69. Kirby JE, Delaney M, Qian Q, Gold HS. Optimal use of Myco/F Lytic and standard BACTEC blood culture bottles for detection of yeast and mycobacteria. Arch Pathol Lab Med 2009;133:93-96.

70. Horvath LL, George BJ, Hospenthal DR. Detecetion of fifteen species of Candida in a automated blood culture system. J Clin Microbiol 2007;45:3062-3064.

71. Horvath LL, Ospenthal DR, Murray CK, Dooley DP. Detection of simulated candidaemia by the Bactec 9240 system with Plus Aerobic/F and Anaerobic/F blood culture bottles. J Clin Microbio 2003;41:4714-4717.

Accepted 30 June 2010 